\title{
Matrix attachment regions included in a bicistronic vector enhances and stabilizes follistatin gene expressions in both transgenic cells and transgenic mice
}

\author{
Xiaoming HU*, Jing GUO*, Chunling BAI, Zhuying WEI, Li GAO, Tingmao HU, Shorgan BOU (凶), \\ Guangpeng LI (ه)
}

Key Laboratory of National Education Ministry for Mammalian Reproductive Biology and Biotechnology/Key Laboratory of Herbivore Reproductive Biotechnology and Breeding of Ministry of Agriculture, Inner Mongolia University, Hohhot 010021, China

\begin{abstract}
In the present study, follistatin (FST) gene expression vectors with either a bicistronic gene transfer cassette alone, or a bicistron gene cassette carrying a matrix attachment region (MAR) were constructed and transfected to bovine fetal fibroblasts. Evaluations of both the integration and expression of exogenous FST indicated that the pMAR-CAG-FST-IRES-AcGFP1-polyA-MAR (pMAR-FST) vector had higher capacity to form monoclonal transgenic cells than the vector without MAR, though transient transfection and integration efficiency were similar with either construct. Remarkably, protein expression in transgenic cells with the pMAR-FST vector was significantly higher than that from the bicistronic vector. Exogenous $F S T$ was expressed in all of the pMARFST transgenic mice at $\mathrm{F}_{0}, \mathrm{~F}_{1}$ and $\mathrm{F}_{2}$. Total muscle growth in $\mathrm{F}_{0}$ mice was significantly greater than in wild-type mice, with larger muscles in fore and hind limbs of transgenic mice. pMAR-FST transgenic mice were also found with more evenly distributed muscle bundles and thinner spaces between sarcolemma, which suggests a correlation between transgene expression-associated muscle development and the trend of muscle growth. In conclusion, a pMAR-FST vector, which excluded the resistant genes and frame structure, enhances and stabilizes FST gene expressions in both transfected cells and transgenic mice.
\end{abstract}

Keywords safety of transgenic, bicistron gene transfer body, transgenic mice, muscle development

Received November 16, 2015; accepted January 23, 2016

Correspondences: gpengli@imu.edu.cn,xurg@cae.cn

${ }^{*}$ These authors contributed equally to the work

\section{Introduction}

Safety issues arising from genetically modified organisms (GMO) are of increasing concern, especially for commercialization of transgenic plants and animals. Generally, recombinant plasmids composed of a backbone sequence, plus resistance and marker genes are used for transgenic purposes. Random integration and inheritance of these elements may generate potential risk factors for the resultant $\mathrm{GMO}^{[1-3]}$. Muller et al. demonstrated that a vector backbone sequence containing a palindrome structure could induce stable secondary structure between plasmids and resulted in illegitimate recombination ${ }^{[2]}$. Philip et al. reported that RNAs transcribed from vector backbone sequences could potentially interfere with the synthesis and processing of RNA from targeted genes. It is likely that vector backbone sequences could cause the occurrence of multiple copies of transgenes ${ }^{[4]}$. Stoger et al. indicated that co-integration of both exogenous vector sequence and the targeted gene sequence could result in formation of giant transgenic loci, which could potentially induce formation of an unstable three-dimensional structure leading to the loss of the locus and the silencing of target genes. Currently, Cre/Loxp, FLP/FRT or R-RS systems are used to delete unnecessary and useless genes $^{[5-7]}$. Although the Cre/Loxp recombination enzyme system can remove resistance or marker genes, this still needs a secondary redundant transfection ${ }^{[7]}$. Using similar recombinant plasmids, plants have been found with linear DNA with transformation, integration and genetic stability, which only contain a promoter and an open reading frame and a terminator ${ }^{[3,4,8]}$. However, linear DNA vectors without a backbone sequence, and selectable and resistant genes have not been used in transgenic animals.

A matrix attachment region (MAR) is a specific type of DNA sequence that widely exist in the boundary sequence of the chromatin loop structure, which may combine with 
the nuclear matrix in vitro ${ }^{[7,9,10]}$. Analysis of the sequence of MARs indicated that all of the known MARs have common features, including $\mathrm{A}+\mathrm{T}$ richness $(>70 \%)$ and sequence motifs, such as the A-box (AATAAAT/CAAA), T-box (TTATTT/ATTT/ATT), autonomously replicating sequence in yeast (ARS), recognition site of Drosophila topoisomerase II, and unwinding DNA for recognition of proteins and curved $\mathrm{DNA}^{[9-11]}$. In plants, MARs are not only important in chromatin folding and regulation of expression of adjacent endogenous genes, but also influence exogenous gene expression, when added on both sides of these genes ${ }^{[9,10]}$. In animals, using expression vectors containing MAR elements could avoid the occurrence of silenced cells, as well as clonal heterogeneity $^{[11]}$. Several MARs are known to enhance protein production in transgenic cells containing human MARs at $\beta$-globin and IFN-loci, or the chicken lysozyme locus ${ }^{[11-13]}$. Therefore, it is important to investigate whether activation of MARs can improve transgene expression in transgenic animals when used as transgene regulators.

Based on previous findings of the positive influence of MAR sequences on integration and expression of exogenous gene in transgenic mice, MAR sequences derived from cattle are hypothesized to have a similar function. In the present study, a vector with a bicistronic gene cassette with MARs added at both ends, but without a bacterial sequence and resistant gene, was constructed as pMAR-CAG-FSTIRES-AcGFP1-polyA-MAR (or pMAR-FST). Its efficacy for transfection, integration and expression were first analyzed in transgenic cells. This pMAR-FST vector was then used to generate transgenic mice. pMAR-FST mice had skeletal muscles that were significantly enlarged when compared to control wild-type mice, suggesting that MAR sequence with FST bicistronic gene-transfer cassette was more appropriate for transgenic applications. To our knowledge, this is the first successful application of bovine MAR sequence along with FST and a bicistronic transfer cassette in transgenic animals.

\section{Materials and methods}

\subsection{Ethics statement}

Experimental procedures were approved by the Animal
Care Commission of the College of Biological Sciences, Inner Mongolia University.

\subsection{Chemicals}

All chemicals and reagents were purchased from SigmaAldrich (St. Louis, MO, USA) unless otherwise stated. Disposable, sterile plastic wares were purchased from Nunclon (Roskilde, Denmark).

\subsection{Construction of vectors}

The correctly sequenced FST gene was first inserted into vector pCAG-IRES-AcGFP1-polyA to construct the vector pCAG-FST-IRES-AcGFP1-polyA. Then two correctly sequenced MAR genes were attached to the vector pCAG-FST-IRES-AcGFP1-polyA, one before the CAG promoter and the other after the polyA signal. This resulted in the vector pMAR-CAG-FST-IRES-AcGFP1-polyAMAR. The bicistronic gene cassette, pCAG-FST-IRESAcGFP1-polyA, was produced by digestion of the plasmid pCAG-FST-IRES-AcGFP1-polyA with restriction enzymes $S a c \mathrm{I}$ and $A f I \mathrm{II}$ followed by separation and extraction using agarose gel electrophoresis. The bicstronic gene cassette with MAR at both ends (pMAR-CAGFST-IRES-AcGFP1-polyA-MAR) was produced from the plasmid pMAR-CAG-FST-IRES2-AcGFP1-polyA-MAR by the same procedure (Fig. 1, Appendix A).

\subsection{Culture and transfection of bovine fetal fibroblast cells}

Bovine fetal fibroblasts were isolated from a 50- to 60-dayold cattle fetus by disaggregation of the body without head and viscera, followed by culturing in Dulbecco's modified Eagle's medium (DMEM; Gibco, Carlsbad, CA, USA) supplemented with $10 \%$ fetal bovine serum (Gibco) at $38.5^{\circ} \mathrm{C}$ in a humidified atmosphere with $5 \% \mathrm{CO}_{2}$. At confluence, the cells were collected by trypsinization for passaging or cryopreservation. The cells were seeded into 12-well plates and cultured in fresh DMEM without antibiotics to achieve $80 \%$ to $90 \%$ of confluence on the day of transfection. The cells were then transfected with Lipofectamine $^{\mathrm{TM}}$ LTX according to the manufacturer's instructions. Briefly, for each well of cationic lipid

(a)

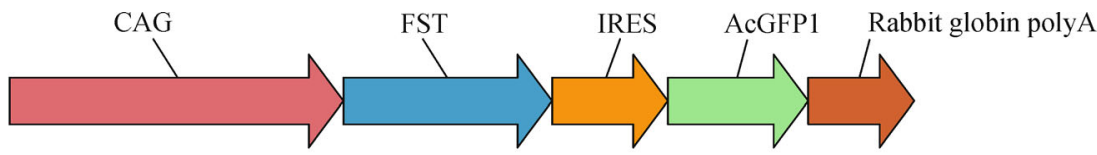

(b)

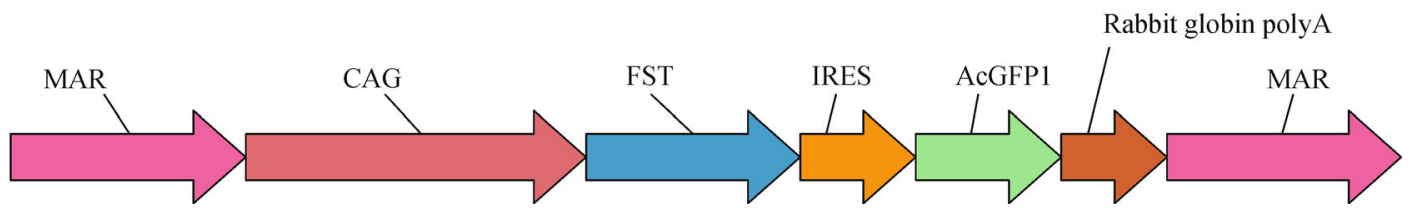

Fig. 1 Construction maps of vectors. (a) Bicistronic gene cassette; (b) pMAR-FST. 
transfection, $800 \mathrm{ng}$ of plasmid DNA $\left(1 \mu \mathrm{g} \cdot \mu \mathrm{L}^{-1}\right)$ was added to $100 \mu \mathrm{L}$ Opti-MEM, mixed thoroughly and then $2 \mu \mathrm{L}$ PLUS reagent added directly to the diluted DNA, mixed gently before incubating at room temperature for $5 \mathrm{~min}$. LTX reagent $(3.5 \mu \mathrm{L})$ was added to $100 \mu \mathrm{L}$ OptiMEM, and then added to the DNA solution, before mixing thoroughly. This mixture was incubated for $30 \mathrm{~min}$ at room temperature. The DNA-lipid complexes were added dropwise to the wells containing cells and gently mixed by rocking the plate back and forth. The cells were incubated at $37^{\circ} \mathrm{C}$ in a $\mathrm{CO}_{2}$ incubator for $6 \mathrm{~h}$, and then changed growth medium.

After culture for $48 \mathrm{~h}$, single cells were sorted to 96-well plates by a flow cytometer for continued culture. The single cells that expressed AcGFP1 were identified as transfectants. After culture for 7 days, single cells were sorted in the same way. The single cells that stably expressed AcGFP1 indicated the exogenous had been integrated into the recipients' chromosome. These single cells were then cultured to form mono-clones. The fluorescence and proportion of positive cells were observed and measured by an inverted fluorescent microscope and flow cytometry, respectively. The negative control group was included to eliminate any error resulting from the instrument.

\subsection{Quantitative real-time PCR}

Total RNA was extracted from each sample using Trizol reagent (Invitrogen, New York, USA), and reverse transcription was performed to generate cDNA using a PrimeScript $^{\mathrm{TM}}$ RT reagent kit (TaKaRa, Dalian, China). The samples were analyzed with SYBR Premix Ex Taq ${ }^{\mathrm{TM}}$ II (TaKaRa). For each sample, target and reference genes were amplified independently on the same plate and in the same experimental run in triplicate. PCR specificity was confirmed by gel electrophoresis on a $2.5 \%$ agarose gel and by the presence of a single peak in the melting curve. For relative quantitative RT-PCR, the amount of target normalized to the reference was calculated by the $2^{-\Delta \Delta C_{T}}$ method.

\subsection{Western blot analysis}

The cells were harvested $48 \mathrm{~h}$ after transfection, rinsed with phosphate-buffered saline and lysed in $150 \mu \mathrm{L}$ of icecold RIPA buffer composed of $50 \mathrm{mmol} \cdot \mathrm{L}^{-1}$ Tris, $150 \mathrm{mmol} \cdot \mathrm{L}^{-1} \mathrm{NaCl}, 0.5 \% \mathrm{Na}$ deoxycholate, $1 \%$ Nonidet P-40 and $0.1 \%$ sodium dodecyl sulfate (SDS). Then, the cell lysates were centrifuged at $4^{\circ} \mathrm{C}$ for $5 \mathrm{~min}$ at $8000 \mathrm{~g}$. The supernatant was electrophoresed in an $8 \%$ SDSpolyacrylamide gel and then transferred onto the polyvinylidene difluoride membrane by performing electroblotting. The membrane was blocked in 5\% non-fat milk in Tris-buffered saline with $0.1 \%$ Tween 20 (TBST) blocking solution at room temperature for $1 \mathrm{~h}$ and subsequently incubated with FLAG-specific monoclonal antibody diluted 1:1000 (F1084, Sigma, USA) in TBST. The membrane was then incubated for $1 \mathrm{~h}$ with horseradish peroxidase-conjugated goat anti-mouse secondary antibody at 1:1000 in TBST, followed by detection using the chemiluminescence labeling detection reagent ECL Plus (Thermo Scientific, Shanghai, China).

\subsection{Monoclonal cells selection and identification}

The sorted single transfected cells were infinitely diluted into $100 \mathrm{~mm}$ dish, to keep cells far apart from each other to guarantee they grew into monoclones, and cultured for $6-9$ days. The mono-clones were selected and passaged in 24well plates. After several passages, the stable cell lines were identified.

The genomic DNA of each cell line was extracted by genome extraction kit and the integration of exogenous genes was detected by PCR. Two pairs of primers were designed to detect the integration of bicstronic of FSTAcGFP1 and MAR-FST. The primers $\left(5^{\prime}-3^{\prime}\right)$ were: FSTAcGFP1, JCF1 sense AACTTCGGGCTTTGCCTCC TGCT, JCR2 and antisense AACTTGCTCATCCAT GCCGTGGG; MAR-FST, JCF1 sense AACTTCGGGC TTTGCCTCCTGCT; and CMAR2 antisense AATCGG TCGACTGAGTCATCCTTTCCTTG.

Due to FST being an endogenous gene expressed in normal bovine fetal fibroblasts, only the co-expression of bicstronic FST-AcGFP1 was detected. Total RNA of each cell line was extracted and tested for the expression of exogenous genes by RT-PCR.

\subsection{Transgenic mice preparation, breeding and identification}

To test if the pMAR-FST vector functions normally at the physiological level, transgenic mice $\left(\mathrm{F}_{0}\right)$ were prepared by pronuclear injection using the pMAR-FST vector. After hybridization of $\mathrm{F}_{0}$ founders with wild-type mice $\mathrm{F}_{1}$ and $\mathrm{F}_{2}$ mice were obtained. At 4-week-old, the tails of the $F_{1}$ and $\mathrm{F}_{2}$ mice were cut to extract genomic DNA, and PCR was performed to determine whether the mice were transgenic. The primers $\left(5^{\prime}-3^{\prime}\right)$ were GF-F sense GCTGGTTATTGTG CTGTCTC and GF-R antisense TCCTGGTCTTCAT CTTCCTC. The total RNA of muscle tissues was extracted from both transgenic and wild-type mice, and reverse transcribed into cDNA to detect the expression of muscle development related genes and the exogenous genes by real-time PCR. Designed primers MSTN, MYoD, MYoG, PAX3, GAPDH, AcGFP and FST are given in Table 1.

\subsection{Animal care and muscle histological analysis}

All experimental mice were maintained under the following conditions: temperature of $25 \pm 1{ }^{\circ} \mathrm{C}$ with $70 \% \pm 4 \%$ relative humidity, automatic light control, rearing density 
Table 1 Primers for real-time PCR analysis

\begin{tabular}{lll}
\hline Gene name & Primers & \multicolumn{1}{c}{ Sequences $\left(5^{\prime}-3^{\prime}\right)$} \\
\hline MSTN & Forward & GCTCAAACAGCCTGAATCCAACTTA \\
& Reverse & CGCAGTCAAGCCCAAAGTCTC \\
MYoD & Forward & TGACCCGTGTTTCGACTCC \\
& Reverse & GCAGGGAAGTGCGAGTGTT \\
MYoG & Forward & CGAGTGCCCCTTGAAGACA \\
& Reverse & CCGACTTCCTCTTACACACCTTACA \\
PAX3 & Forward & GTCCCATGGCTGCGTCTCTAA \\
& Reverse & TCTCCACGTCAGGCGTTGTC \\
GAPDH & Forward & AAATGGTGAAGGTCGGTGTGAAC \\
AcGFP & Reverse & CAACAATCTCCACTTTGCCACTG \\
FST & Forward & ATGGCAACATCCTGGGCAATAAGAT \\
& Reverse & CGCCGATGGGGGTATTCTGCTGGTA \\
\hline
\end{tabular}

less than or equal to four per cage. Mice were provided food and water ad libitum. All mice were anesthetized before being euthanized.

Small sections of muscle tissue were taken from the forelimb and hindlimb of both $\mathrm{F}_{0}$ transgenic and wild-type mice. The tissues were fixed in $4 \%$ paraformaldehyde, and embedded in paraffin. Five-micrometer-thick sections were deparaffinized and stained with hematoxylin-eosin and pictures were taken from four random fields at $400 \times$ magnification under a microscope (DS-Ri1, Nikon) (http:// imagej.net) was used to calculate the muscle crosssectional area.

\subsection{Statistical analysis}

Each experiment was performed at least three times. All data were analyzed using SPSS 20.0 statistical software (IBM Corporation, Somers, NY, USA). Data were tested by one-way ANOVA and least-significant difference tests, and reported as the mean and SEM. For all analyses, $P<0.05$ was considered significant.

\section{Results}

\subsection{Transfection and integration efficiency}

Results from assays of both fluorescence microscopy and flow cytometry indicated that there was no significant difference in the transient transfection efficiency between the bicistronic gene cassette (17.4\%) and pMAR-FST $(21.0 \%)$ (Table 2). In addition, there was also no significant difference in integration rate between the bicistronic gene cassette $(0.6 \%)$ and pMAR-FST (1.3\%) (Table 3$)$.

3.2 Comparison of FST RNA expressions between transgenic cells with bicistronic or pMAR-FST gene cassette

cDNAs from FST mRNA were generated from transgenic cells with the bicistronic cassette, pMAR-FST and negative control, in order to detect the FST mRNA expression by Real Time PCR; $\beta$-actin was as the internal control. As shown in Fig. 2, setting the negative control group to 1, the level of FST expression in pMAR-FST was 19.4,

Table 2 Comparison of transfection efficiency

\begin{tabular}{lcccc}
\hline Experimental repeats & 1 & 2 & 3 & Mean \\
\hline Bicistronic gene cassette/\% & 19.31 & 11.83 & 21.03 & $17.39 \pm 3.99$ \\
pMAR-FST/\% & 23.16 & 14.07 & 25.93 & $21.05 \pm 5.06$ \\
Negative control/\% & 0.15 & 0.24 & 0.12 & 0.17 \\
\hline
\end{tabular}

Table 3 Comparison of the integration efficiency

\begin{tabular}{lcccc}
\hline Experimental repeats & 1 & 2 & 3 & Mean \\
\hline Bicistronic gene cassette/\% & 0.85 & 0.39 & 0.59 & $0.61 \pm 0.19$ \\
pMAR-FST/\% & 1.36 & 1.48 & 1.18 & $1.34 \pm 0.12$ \\
Negative control/\% & 0.04 & 0.06 & 0.06 & 0.05 \\
\hline
\end{tabular}




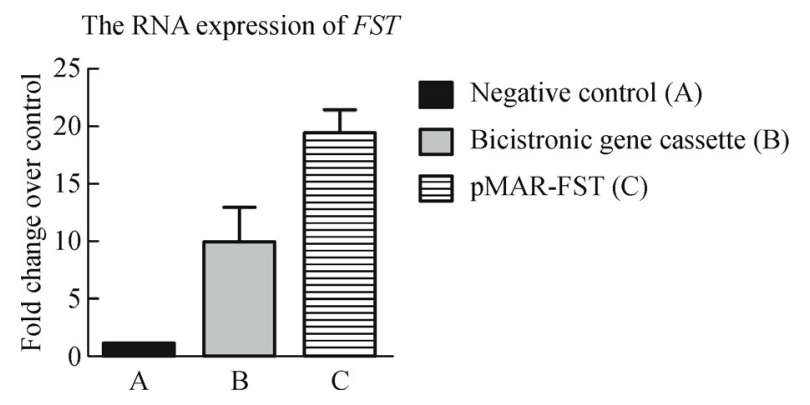

Fig. 2 FST mRNA expression by real-time PCR

significantly higher than the 7.35 obtained with the bicistronic cassette.

Together, these results indicated that pMAR-FST enhanced FST expression compared to the bicistronic construct.

3.3 Comparison of FST protein expressions in transgenic cells with either the bicistronic gene or pMAR-FST constructs

The FST expression with the bicistronic gene construct was similar to the negative control. However, the pMARFST transfected cells had significantly higher expression of exogenous FST when compared to the bicistronic gene construct transfected cells or the controls. No significant difference in GAPDH expression was observed between the different groups. Together, these results suggested that the MAR sequence may increase the expression of exogenous FST genes. The mechanism, however, needs further study (Fig. 3).
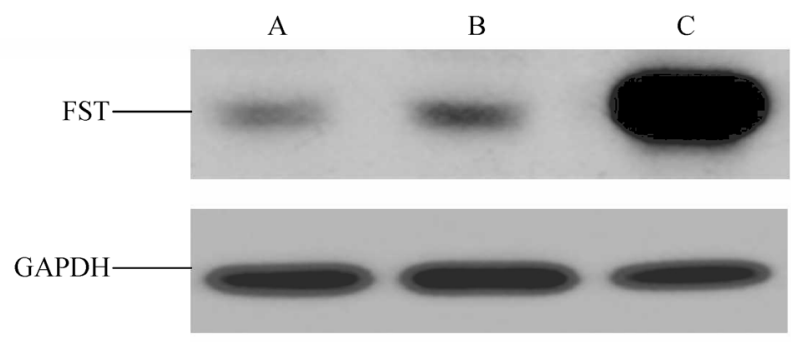

Ratio of FST/GAPDH $\quad 0.59$

0.66

1.56

Fig. 3 FST translation in bovine fetalblasts. FST protein after translation was detected by western blot assay. The mean grave value was measured by using Imagej software. A, The negative control; B, bicistronic gene cassette; C, pMAR-FST vector.

\subsection{Selection and characterization of monoclonal transgenic cells}

\subsubsection{Selection of monoclonal cells}

To build a secure gene transfer cassette that did not contain resistance genes or other additional frame sequence, we analyzed the transgenic cells by standard antibiotics screening methods after transfection. In the pMAR-FST group, positive clones formed in 5 days after confection, while the monocloned cells were observed until 9 days after confection with the linear vector. A total of 23 monoclones in the pMAR-FST group were obtained and eight of them were transgenic. Four monoclones were obtained from the group containing the bicistronic gene construct and one of them was transgenic. The clones from the pMAR-FST vector were larger and grew more vigorously than those from clones with bicistronic gene cassette vector (Fig. 4).

\subsubsection{Characterization of the integration of FST-AcGFP1 and FST-MAR sequences in transgenic cells}

To determine whether the whole pMAR-FST vector was integrated into the genome, sequences from FST-AcGFP1 and FST-MAR were examined. Genomic DNA of eight transgenic monoclonal cells were extracted and tested by PCR. The results showed that FST-AcGFP1 had been integrated in all eight of the transgenic cell lines (Fig. 5) and that FST-MAR had been integrated in seven of the eight transgenic cell lines (Fig. 5), which indicated that seven of the eight transgenic monoclonal cells had the pMAR-FST fully integrated into genome of host cells.

\subsection{3 mRNA expression in the transgenic cells}

The mRNAs were isolated from eight pMAR-FST transgenic cells and then copied into cDNA. The level of FST mRNA expression was detected by real-time PCR, $\beta$ actin was used as the internal control. The results showed that FST-AcGFP1 was expressed in all eight transgenic cell lines (Fig. 6).

\subsection{Characterization of transgenic mice}

\subsubsection{Breeding of transgenic mice}

Three founder $\left(\mathrm{F}_{0}\right)$ pMAR-FST transgenic mice were obtained consisting of two males and one female. The $\mathrm{F}_{0}$ male mice were mated with wild-type C57BL/6J female mice and four heterozygous $F_{1}$ and four $F_{2}$ transgenic mice were obtained (half siblings) (Fig. 7). The mRNAs were isolated from all of the $F_{0}, F_{1}$ and $F_{2}$ pMAR-FST transgenic mice and reverse transcribed into cDNA to detect mRNA expression of exogenous genes by real-time PCR. The results showed that the exogenous genes were expressed in all of the pMAR-FST transgenic mice (Fig. 8).

\subsubsection{Expression of genes associated with muscle} development

To analyze whether the expressions of muscle development 

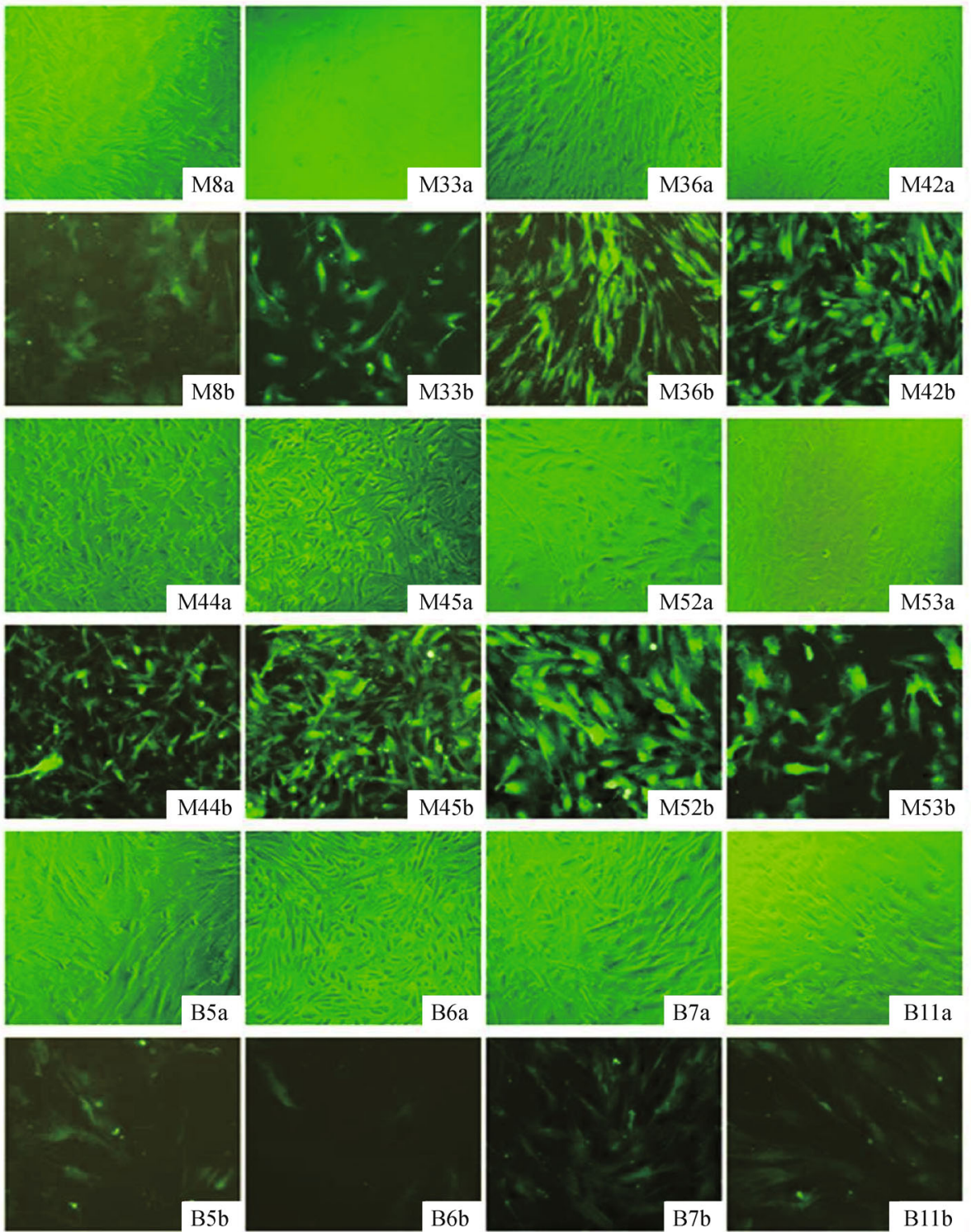

Fig. 4 Transgenic cell monoclones obtained from different vectors. a, Growth state of transgenic cells under the bright-field; b, fluorescence levels of transgenic cells under the dark field. M8, M33, M36, M42, M44-45, M52-53, Monoclone of transgenic cells with pMAR-FST vector; B5-7, B11, monoclone of transgenic cells with bicistronic gene cassette.

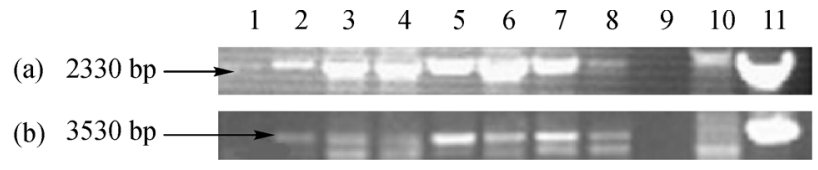

Fig. 5 PCR analysis of FST-AcGFP1 (a) and FST-MAR integration (b). Lanes assigned as: 1, M8; 2, M33; 3, M36; 4, M42; 5, M44; 6, M45; 7, M52; 8, M53; 9, control of $\mathrm{ddH}_{2} \mathrm{O}$; 10 , negative control; 11 , positive control.

related genes changed in $\mathrm{F}_{0}, \mathrm{~F}_{1}$ and $\mathrm{F}_{2}$ pMAR-FST transgenic mice, MSTN (myostatin), MYoG, MYoD and $P A X 3$ were each assayed by real-time PCR. The results

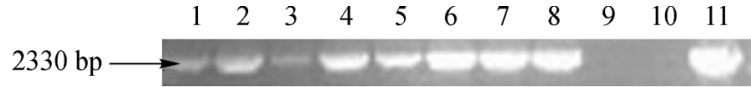

Fig. 6 RT-PCR analysis on RNA expressions.1, M8; 2, M33; 3, M36; 4, M42; 5, M44; 6, M45; 7, M52; 8, M53; 9, control of ddH20; 10, negative control; 11, positive control.

showed that MSTN was upregulated, while $M Y o G, M Y o D$ and $P A X 3$ were downregulated in the $\mathrm{F}_{0}$ pMAR-FST transgenic mice in comparison with the wild-type mice. The expression of MSTN, MYoG, MYoD and PAX3 were all downregulated in $\mathrm{F}_{1}$ and $\mathrm{F}_{2}$ pMAR-FST transgenic mice (Fig. 9). 


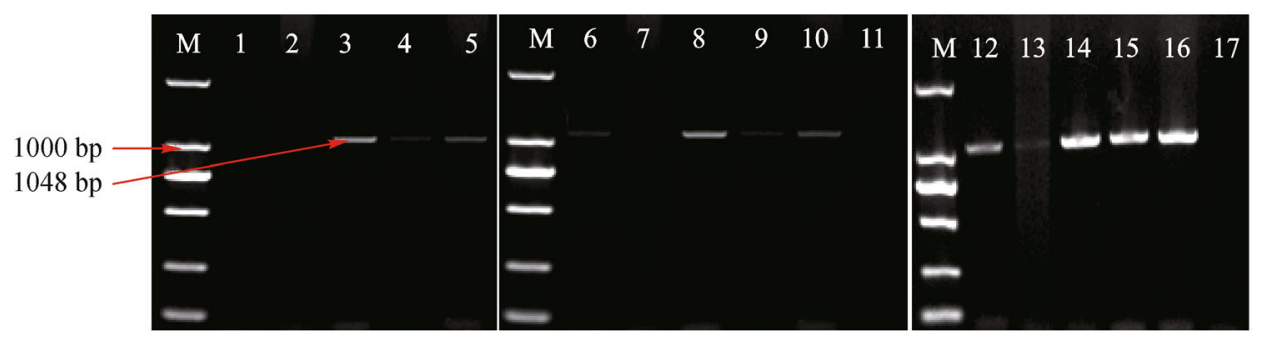

Fig. 7 PCR analysis on FST gene in transgenic mice. M, DL2000 Marker; 1, 2, 7, 11, 17, the control of dd $\mathrm{H}_{2} \mathrm{O}$; 3-5, the primary generation of transgenic mice; 6, 8-10, the first generation of transgenic mice; 12-15, the second generation of transgenic mice; 16, the plasmid of positive control.
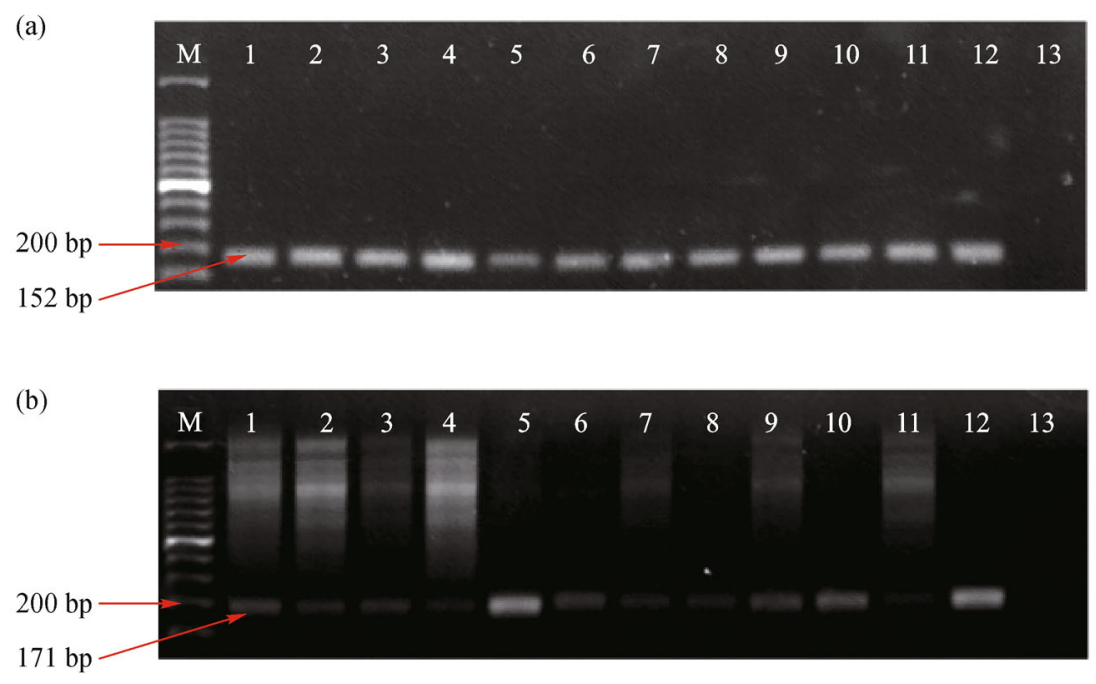

Fig. 8 RT-PCR analysis on exogenous genes expressions. (a) RT-PCR analysis of FST expression; (b) RT-PCR analysis of $A c G F P$ expression. M, 100bp Marker; 1-3, primary generation of transgenic mice; 4-7, the first generation of transgenic mice; 8-11, the second generation of transgenic mice; 12, plasmid of positive control; 13, control of $\mathrm{ddH}_{2} \mathrm{O}$.

(a)

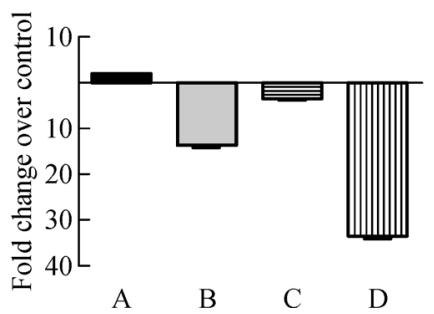

(b)

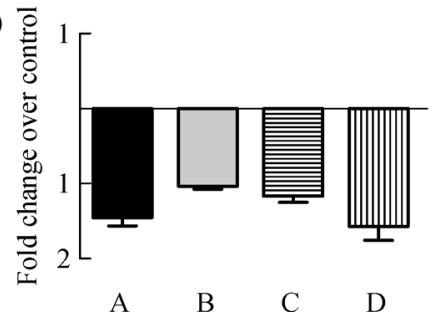

(c)

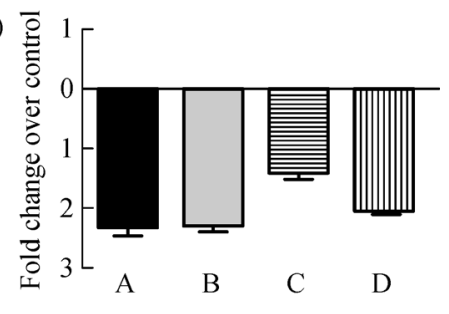

Fig. 9 Expressions of FST enhanced muscle development in transgenic mice. The fold changes of muscle development related gene between FST transgenic mice and wild-type mice were calculated from the results after qRT-PCR assays. (a) $\mathrm{F}_{0}$ transgenic mice; (b) $\mathrm{F}_{1}$ transgenic mice; (c) $\mathrm{F}_{2}$ transgenic mice. A, MSTN; B, MyoG; C, MyoD; D, PAX3.

3.5.3 FST transgenic mice induced enhanced muscle development

Anatomical analysis showed that the whole body lengths of the $\mathrm{F}_{0}$ pMAR-FST transgenic mice were similar to the wild-type mice, both being $110 \mathrm{~mm}$ (Fig. 10a). However, the fore and hind limbs of pMAR-FST transgenic mice were 24 and $30 \mathrm{~mm}$, respectively, which were longer than the wild-type mice (17 and $23 \mathrm{~mm}$, respectively). The bodyweight of pMAR-FST transgenic mice were $49 \mathrm{~g}$, which was heavier than the wild-type mice at $42 \mathrm{~g}$ (Fig. 10b). 
Histological analysis revealed that the muscle bundles were distributed more evenly, and the inter-space between myofibers in pMAR-FST transgenic mice (Fig. 10c) were thinner than that in wild-type mice (Fig. 10d). The average myofiber diameter was $4510 \mu^{2}$ in pMAR-FST transgenic mice, which significantly larger than wild-type mice with an average of $2740 \mu \mathrm{m}^{2}$.

\section{Discussion}

A MAR is a fragment of DNA sequence that can specifically combine with the nuclear matrix and anchor DNA or chromatin to $\mathrm{it}^{[10]}$. As natural parts of the eukaryotic genome, MAR sequences act as the boundary elements which isolate protect individual genes from the influence of some control elements in adjacent areas. Argyros et al. reported that development of S/MAR minicircles enhanced persistent expression of a transgene in mouse liver ${ }^{[14]}$. In the present study, bovine MAR sequence was isolated from the bovine genome and added to both sides of an FST bicistronic gene construct from which the resistant gene and bacterial frame structure had been deleted. The FST bicistronic gene construct with MAR was used for delivery into both mouse fetal fibroblasts and fertilized oocytes for generating transgenic mice. Similar transfection efficiencies were shown using both MAR-added and classic MAR-free vectors, which indicated that deletion of bacterial sequence did not affect transfection. Moreover, using pMAR-FST vector resulted in significantly higher expression levels of both mRNA and protein than using MAR-free vector as controls. Compared with the results with the control vector, the monoclonal cells with MAR-mediated vector formed more rapidly and were larger in size. Bicistronic gene constructs carrying MARs formed positive clones more rapidly, which may be because the MAR sequence can more effectively change the cell state and promote cell proliferation, so that the expression of transgenes tends to be stabilized. Previous studies have confirmed that MAR sequence can enhance the expression level of an exogenous gene and the expressions tends to be more stable ${ }^{[13]}$. These results suggest that the use of MAR sequences improves both exogenous gene integration and expressions of mRNA and protein, as well as the efficiency of formation of monoclonal cells.

FST is known to be a gene for upregulation of muscle growth, promoting muscle development through the prevention of MSTN binding to its receptor ${ }^{[15]}$. Previous studies have shown that mice with the FST homozygous mutation resulted in significantly decreased muscle development ${ }^{[15-17]}$. In the pMAR-FST transgenic mice, FST as exogenous gene was detectable in DNA, RNA and protein of all $\mathrm{F}_{0}, \mathrm{~F}_{1}$ and $\mathrm{F}_{2}$ mice, which demonstrated that the backbone sequences-free MAR-bicistronic-gene-transfer cassette could integrate into the genome and passes from generation to generation. Gene expression associated with muscle development was consistent with the tendency of muscle physiological growth with FST overexpression in all of $\mathrm{F}_{0}, \mathrm{~F}_{1}$ and $\mathrm{F}_{2}$ transgenic mice. MSTN expression was (a)

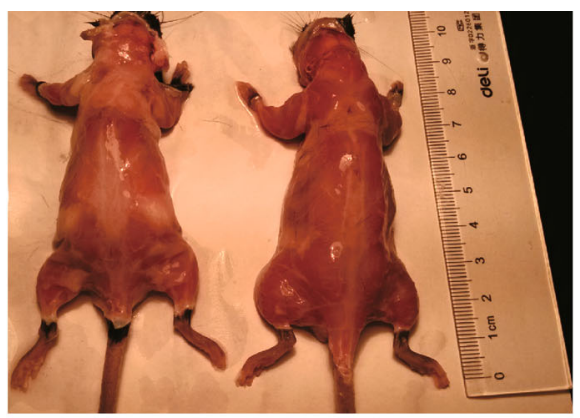

(c)

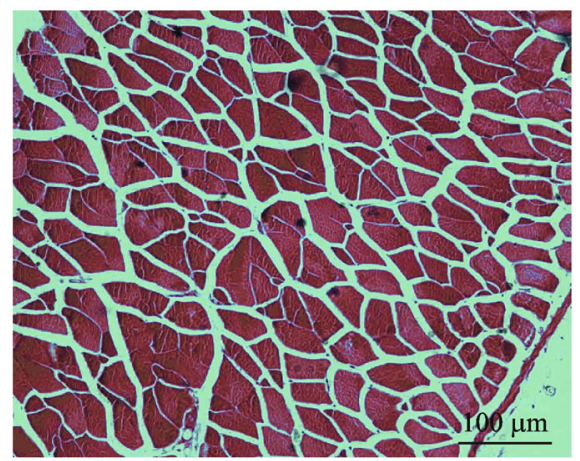

(b)

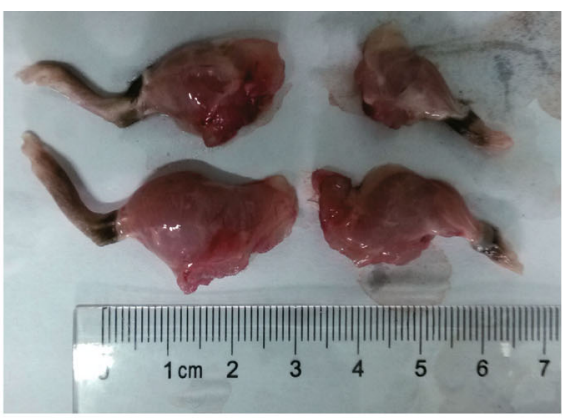

(d)

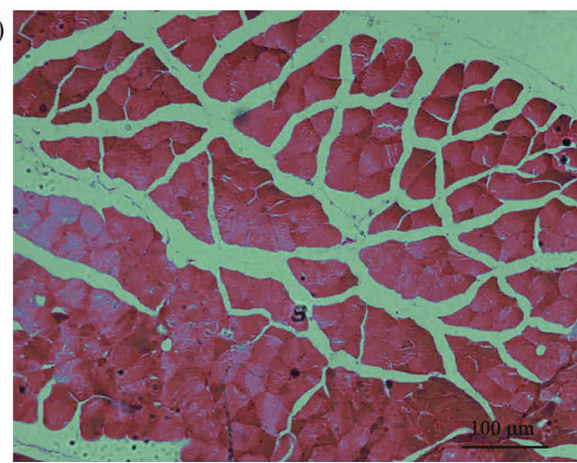

Fig. 10 FST transgenic mice induced strong muscle development with the increased muscle bundles. (a) The body length of transgenic mice (left) and wild-type mice (right); (b) the limbs of transgenic mice (left) and wild-type mice (right); (c) histological analysis on muscle tissues from primary generation of transgenic mice; (d) histological analysis on muscle tissues from primary generation of wild-type mice. 
upregulated, while the expressions of $M Y o G, M Y o D$ and $P A X 3$ were all downregulated in the $\mathrm{F}_{0}$ transgenic mice. In addition, expression of MSTN, MYoG, MYoD and PAX3 were all downregulated in both $\mathrm{F}_{1}$ and $\mathrm{F}_{2}$ transgenic mice. The upregulation of MSTN in $\mathrm{F}_{0}$ transgenic mice probably reflected the compensation due to overexpression of $F S T^{[18]}$. The downregulation of MSTN in $\mathrm{F}_{1}$ and $\mathrm{F}_{2}$ transgenic mice probably shows that a functional balance of FST and MSTN can be established, and MSTN can be downregulated ${ }^{[18]}$. All the other genes examined, including $M Y o G, M Y o D$ and $P A X 3$, were also downregulated, suggesting the same effect of FST overexpression in all generations of transgenic mice.

In the present study, FST overexpression resulted in increased muscle mass growth in transgenic mice (Fig. 10). The limb weight and muscle mass in transgenic mice significantly increased in comparison to the wild-type mice. The results of histological analysis showed that the average myofiber diameters in FST overexpressing mice were significantly larger than that in wild-type mice (Fig. 10). These phenomena were most likely similar to the findings with $M S T N$-mutated mice ${ }^{[19,20]}$. This suggests that the FST gene carried by MAR sequence can be expressed effectively in the individual and play corres-ponding functions.

\section{Conclusions}

To our knowledge, this is the first study using MAR sequences to regulate FST for the delivery of exogenous genes in both transgenic cells and transgenic animals. Results indicated that the MAR with an FST bicistronic gene cassette not only stabilized integration, but also enhanced the expression of the exogenous FST gene in both transgenic cells and transgenic mice.

Acknowledgements Thanks to Dr. Xin Wang, Adjunct Professor of Inner Mongolia University, for his help during preparation of this manuscript. Thanks to Dr. Yang Yang and Lei Yang for their technical assistance. This work was supported by the National Transgenic Breeding Project (2014ZX08007-002).

Supplementary materials The online version of this article at http://dx.doi.org/ 10.15302/J-FASE-2016087 contains supplementary material (Appendix A).

Compliance with ethics guidelines Xiaoming Hu, Jing Guo, Chunling Bai, Zhuying Wei, Li Gao, Tingmao Hu, Shorgan Bou, and Guangpeng Li declare that they have no conflict of interest of interest or financial conflicts to disclose.

All applicable institutional and national guidelines for the care and use of animals were followed.

\section{References}

1. Kobelt D, Schleef M, Schmeer M, Aumann J, Schlag P M, Walther W. Performance of high quality minicircle DNA for in vitro and in vivo gene transfer. Molecular Biotechnology, 2013, 53(1): 80-89
2. Müller A E, Kamisugi Y, Gruneberg R, Niedenhof I, Hörold R J, Meyer P. Palindromic sequence and A + T-rich DNA elements promote illegitimate recombination in Nicotiana tabacum. Journal of Molecular Biology, 1999, 291(1): 29-46

3. Stoger E, Williams S, Keen D, Christou P. Molecular characteristics of transgenic wheat and the effect on transgene expression. Transgenic Research, 1998, 7(6): 463-471

4. Fu X D, Duc L T, Fontana S, Bong B B, Tinjuangjun P, Sudhakar D, Twyman R M, Christou P, Kohli A. Linear transgenic constructs lacking vector backbone sequences generate low-copy-number transgenic plants with simple integration patterns. Transgenic Research, 2000, 9(1): 11-19

5. Chen Z Y, Yant S R, He C Y, Meuse L, Shen S, Kay M A. Linear DNAs concatemerize in vivo and result in sustained transgene expression in mouse liver. Molecular Therapy, 2001, 3(3): 403410

6. Matake M A, Mette M F, Matzke A J. Transgene silencing by the host genome defense: implications for the evolution of epigenetic control mechanisms in plants and vertebrates. Plant Molecular Biology, 2000, 43(2-3): 401-415

7. Harraghy N, Gaussin A, Mermod N. Sustained transgene expression using MAR elements. Current Gene Therapy, 2008, 8(5): 353-366

8. Breitler J C, Labeyrie A, Meynard D, Legavre T, Guiderdoni E. Efficient micro projectile bombardment-mediated transformation of rice using gene cassettes. Theoretical and Applied Genetics, 2002, 104(4): 709-719

9. Bode J, Benham C, Knopp A, Mielke C. Transcriptional augmentation: modulation of gene expression by scaffold/matrix attached regions (S/MAR elements). Critical Reviews in Eukaryotic Gene Expression, 2000, 10(1): 73-90

10. Harraghy N, Buceta M, Regamey A, Girod P A, Mermod N. Using matrix attachment regions to improve recombinant protein production. Methods in Molecular Biology, 2012, 801: 93-110

11. Girod P A, Nguyen D Q, Calabrese D, Puttini S, Grandjean M, Martinet D, Regamey A, Saugy D, Beckmann J S, Bucher P, Mermod N. Genome-wide prediction of matrix attachment regions that increase gene expression in mammalian cells. Nature Methods, 2007, 4(9): 747-753

12. Kim J M, Kim J S, Park D H, Kang H S, Yoon J, Baek K, Yoon Y. Improved recombinant gene expression in $\mathrm{CHO}$ cells using matrix attachment regions. Journal of Biotechnology, 2004, 107(2): 95105

13. Zahn-Zabal M, Kobr M, Girod P A, Imhof M, Chatellard P, deJesus M, Wurm F, Mermod N. Development of stable cell lines for production or regulated expression using matrix attachment regions. Journal of Biotechnology, 2001, 87(1): 29-42

14. Argyros O, Wong S P, Constantinos F, Tolmachov O, Waddington S N, Howe S J, Niceta M, Coutelle C, Harbottle R P. Development of $\mathrm{S} / \mathrm{MAR}$ minicircles for enhanced and persistent transgene expression in the mouse liver. Journal of Molecular, 2011, 89(5): 515-529

15. Nakatani M, Takehara Y, Sugino H, Matsumoto M, Hashimoto O, Hasegawa Y, Murakami T, Uezumi A, Takeda S, Noji S, Sunada Y, Tsuchida K. Transgenic expression of a myostatin inhibitor derived from follistatin increases skeletal muscle mass and ameliorates dystrophic pathology in mdx mice. FASEB Journal, 2008, 22(2): $477-487$ 
16. McPherron A C, Lawler A M, Lee S J. Regulation of skeletal muscle mass in mice by a new TGF- $\beta$ superfamily member. Nature, 1997, 387(6628): 83-90

17. Bodnár D, Geyer N, Ruzsnavszky O, Oláh T, Hegyi B, Sztretye M, Fodor J, Dienes B, Balogh Á, Papp Z, Szabó L, Müller G, Csernoch L, Szentesi P. Hypermuscular mice with mutation in the myostatin gene display altered calcium signaling. Journal of Physiology, 2014, 592(6): 1353-1365

18. Kocamis H, Gulmez N, Aslan S, Nazli M. Follistatin alters myostatin gene expression in $\mathrm{C} 2 \mathrm{C} 12$ muscle cells. Acta Veterinaria Hungarica, 2004, 52(2): 135-141

19. Szabó G, Dallmann G, Müller G, Patthy L, Soller M, Varga L. A deletion in the myostatin gene causes the compact (Cmpt) hypermuscular mutation in mice. Mammalian Genome, 1998, 9 (8): 671-672

20. Lee S J, McPherron A C. Regulation of myostatin activity and muscle growth. Proceedings of the National Academy of Sciences of the United States of America, 2001, 98(16): 9306-9311 